\title{
Dyadic facial affective indicators of severity of symptomatic burden in patients with
} panic disorder ${ }^{1}$

\author{
Cord Benecke* \& Rainer Krause $^{+}$ \\ * Department of Psychology, University of Innsbruck \\ ${ }^{+}$Clinical Psychology and Psychotherapy, University of the Saarland
}

Running head: Facial affective indicators of severity

Corresponding Author:

Prof. Dr. Cord Benecke

Department of Psychology

University Innsbruck

Innrain 52

6020 Innsbruck, Austria

Tel: 0043 (0) $512-5075575$

Fax: 0043 (0) $512-5072835$

E-mail: cord.benecke@uibk.ac.at

\footnotetext{
${ }^{1}$ The present results are part of the Project "Relationship Regulation and Therapy Success in the Psychotherapy of Patients with Panic Disorders”, supported by Deutsche Forschungsgemeinschaft.
} 


\section{Abstract}

Background: Based on the results of research on facial affective behaviour in different psychological disorders, as well as on available findings on the specific behaviour of patients with panic disorder in interaction with their therapists, hypotheses about dyadic facial affective behaviour and its correlation with symptomatic burden of female panic patients are formulated.

Sampling and Methods: The facial affective behaviour of 20 patients with panic disorder and their therapists, coded with EMFACS, in the first treatment session is analyzed regarding interactive enmeshment, and for a subgroup of 15 dyads these data are correlated with those on symptomatic burden before treatment.

Results: A high degree of interactive enmeshment between patient and therapist correlates positively with the severity of symptomatic burden. All dyadic enmeshment indicators show highly significant positive correlations with body-related symptoms, but not with more general variables like global severity index of the SCL-90R or general anxiety (STAI).

Conclusions: These results are discussed against the background of the specific psychodynamic of panic patients and show that, on the one hand, therapists practise an interactive abstinence, but on the other hand, they tend to be pulled into a specific interactive enmeshment by patients with greater symptomatic burden. Limitations of the study arise from the small sample and the lack of a comparison-group, therefore the question if the results are disorder-specific or more general cannot be answered.

Keywords: panic disorder - dyadic facial affective behaviour - severity of disorder 


\section{Introduction}

Emotions play a central role in psychopathology. In the last few years, the idea of basic or primary affects has gained increasing recognition in the field of emotion research [1, 2]. Ekman [1] postulates anger, fear, sadness, happiness, disgust and surprise as culturally invariant, innate and thus primary emotions; usually also contempt [2, 3] is assumed to be a primary emotion. Each primary affect can be characterised by a specific trigger configuration, i.e. a certain subjective evaluation of a situation and/or an object, a specific action-tendency, a specific wish. Furthermore, each affect is assumed to correspond with a specific facial affective expression, which serves important relationship-regulating functions [2, 4].

Several studies have shown that patients with mental disorders differ in their facial affective behaviour from normal controls; in interaction with healthy controls or clinical interviewers, individuals with mental disorders show a generally reduced facial affective activity, with less positive facial expression and more negative affects. Additionally, the interaction partners made a specific adaptation to the patients' facial affective behaviour, which in turn serve to maintain the underlying maladaptive interaction patterns of the patients $[5,6,7,8,9,10,11]$. If emotion regulation is determined by the interaction partner included, it is called interactive emotion regulation. Clinically, the use of interactive emotion regulation strategies is usually linked with structural disorders $[2,12,13]$. Facial affective behaviour has an essential role to play in this kind of emotion regulation. In case of "successful" interactive regulation, the actual interaction allows both partners to regulate their own emotions in "cooperation" with the other.

Schwab [9] showed that interactions between severely disturbed patients (schizophrenia; Colitis Ulcerosa) and healthy persons are characterized by specific reciprocal smiling patterns: the patients responded to the "felt" smile of their healthy interaction partners significantly more often with a "social” smile. The patients did not react in a totally impolite 
manner since they smiled back in order to keep up the social relationship to some extent; at the same time, however, they established a clear emotional distance. Thus, within a very short time, dyadic regulation patterns were established in these dyads, which enabled both interaction partners to continue the interaction with reduced involvement [9; see also 7, 8].

Also the study of Merten [14] indirectly indicate a connection between interactive regulation and disorder severity: facial affective "enmeshment" between patient and therapist, as a possible marker of disorder severity, correlated with poor treatment outcome. Additional evidence supporting this assumed link between interactive regulation and disorder severity can be derived from the studies of Rasting \& Beutel [15] and Rasting et al. [11].

In general, therapists show reduced frequencies of facial affective behaviour compared with their patients, which is considered as a manifestation of interactive abstinence; but this abstinence is only a relative one: therapists do show facial affects, including negative, which has been understood as an acting out of counter-transference [e.g. 14; 15]

So far there are no studies which relate indicators of interactive enmeshment with measures of disorder severity within a homogeneous diagnostic group. The present study investigates this issue in a group of female patients with panic disorders.

\section{Facial affective relationship-offers of patients with panic disorder}

The psychodynamic of patients with panic disorder is essentially dominated by a dependenceautonomy-conflict, connected with a kind of anxious clinging relationship; however, it is accompanied by subconscious ambivalences [e.g. 16, 17]. The separation issue, real or fantasized separations and losses of important objects, is assumed to be a key trigger of panic attacks. The absence of a positive self-regulation competence is to be compensated by the relationship-partners, who are functionalized as "steering objects" [18]. The wish for independence and autonomy cannot be realized, because patients are afraid to take the risk of 
loosing bonding they needed. Autonomy impulses or expression of annoyance or rages, even if unconscious, inevitably lead patients to imagine that they will be abandoned by these objects. As a result, unconscious aggressive impulses serve as frequent symptom-triggers. In three single case studies of patients with anxiety disorder (one male, two females) [2, 14, 19] similar behavioural pattern were found, consisting of frequent smiles and very rarely negative affect expressions on the part of the patients. Based on these results and the described dependence of panic patients, Benecke \& Krause [20] expected the facial affective behaviour of patients with panic disorder to be dominated by Duchenne smile (the so-called "felt" smile), accompanied by a rather limited affective repertoire, in particular, by a reduction in aggressive facial expression, reflecting their difficulty in striving for separation and autonomy. As expected, the facial affective behaviour of the patients studied was dominated by smiling, but it proved to be quite heterogeneous as well: the facial expressions of not every patient was characterised by the expected Duchenne smiles. They were not the leading affective expression: some patients showed considerably aggressive facial expressions [20]. The following investigation of the connection between facial affective behaviour and the severity of symptomatic burden is based on the same sample as of the previous study [20]. It now includes dyadic measures of facial affective interaction between patients and therapists.

\section{Hypotheses}

\section{Hypothesis 1: Interactive abstinence of the therapists}

In general, psychodynamic therapists are supposed to practise an abstinent interactive behaviour towards their patients, but this abstinence is not absolute. Therefore, we assume that repetitive sequence patterns at the micro level of the dyadic facial affective behaviour indicating interactive "enmeshment" will be found. According to the described psychodynamic of panic patients, particularly dyadic patterns consisting of Duchenne smiles 
are to be expected, with patients responding to the Duchenne smiles of the therapists with a Duchenne smile more frequently than the other way round, which reflects the interactive abstinence of the therapists.

\section{Hypothesis 2: Interactive "enmeshment" and symptomatic burden}

Regarding the connection between dyadic sequence patterns and symptomatic burden we assume the following: The number of dyadic repetitive sequence patterns and their frequency of occurrence positively correlate with the severity of symptomatic burden. This means that more severely disturbed patients increasingly tend to "enmesh" their interaction partners, thus also their therapists, in the sense of an interactive affect regulation strategy.

\section{Method}

\section{Sample}

The total sample of the study consisted of 20 therapeutic dyads: 20 female patients with ICD10 codes F40.01 (agoraphobia with panic disorder: six patients) and F41.0 (panic disorder: 14 patients) according to Hiller et al. [21], and their individual therapists. The therapists were 13 experienced, male therapists trained in psychoanalysis, their treatment-technique based on the "Manual of Panic Focussed Psychodynamic Psychotherapy" [16]. Seven of the therapists treated one patient each, five of the therapists treated two patients each, and one therapist treated three patients. The mean of treatment sessions was 28.3. The patients were between 23 and 57 years of age (mean $=35.2$ years) and suffered from anxiety disorder for an average of 5.2 years $(\mathrm{SD}=6.14$; with a range between 6 weeks and 20 years).

Each first therapy-session was videotaped, and the facial affective behaviour of patients and therapists was coded with EMFACS. Since the questionnaires were not completed by all of 
the patients of the study, the sample used for testing hypothesis 2 is reduced to 15 and 13 dyads, respectively.

\section{Questionnaires:}

The following questionnaires were administered to the patients before treatment: SCL 90-R Symptom check list [22]; FBL-R- Freiburger Beschwerde Liste [23] which records the presence and extent of physical complaints on nine subscales and one total score; STAI - State Trait Anxiety Inventory [24]; BSQ (Body Sensation Questionnaire) to measure fear of physical symptoms [25], and ACQ (Agoraphobic Cognitions Questionnaire) to measure fearrelated cognitions [25] with the two subscales "physical crises" and "loss of control" as well as a total score. The total scores of the questionnaires are taken as criteria for severity of symptomatic burden.

\section{Coding of facial affective behaviour}

The facial affective behaviour was coded with the Emotional Facial Action Coding System (EMFACS, [26]). EMFACS is a short form of FACS (Facial Action Coding System, [27, 28]) and concentrates on emotion-relevant facial movements. The coding of facial behaviour concentrates on visible activation of facial muscles (action units, AUs) ${ }^{2}$. With a so-called "lexicon", facial expression codings are assigned to affect categories: anger, disgust, contempt, fear, sadness, surprise and smile. In addition, there is a set of rules which permits distinguishing between "felt" joy expressions (Duchenne smile; AUs 6+12) and "social" smiles (AU 12 only). The use of EMFACS enables gathering data on the frequency and the temporal placement of different facial affective signals of patient and therapist.

\footnotetext{
${ }^{2}$ For being authorized as a coder, the so called "final test" has to be passed, in which the interrater-reliability is proved by the Human Interaction Laboratory in San Francisco. In a recent study concerning interrater-reliability of FACS-Codings, good to very good values were found [31].
} 
Since the duration of the individual sessions varied (mean $=47.3$ minutes, $\mathrm{SD}=5.1$ ), the data on frequencies of affective facial behaviors were adjusted to conform to a 50-minute session.

\section{Detecting repetitive sequence patterns - "interactive enmeshment"}

For analysing repetitive sequence patterns of facial affective behaviour pattern detection software, called THEME [29, 30], was used. THEME scans a behavioural stream for repetitive event successions, taking the time intervals between events into consideration. To be recognized as a repetitive pattern, the events (here facial affective behaviours) have to appear in a repetitive sequence at similar time intervals, whereby the patterns are tested against a chance-distribution for statistical significance. The null hypothesis assumes that all traceable events and also all time intervals between them are distributed randomly over the observation period. The empirical event succession is examined against this null-hypothesis for significance. Monadic and dyadic patterns are differentiated. Monadic patterns describe repetitive successions within the behaviour of one person. Dyadic patterns describe repetitive successions of interactive behaviours between patient and therapist. A frequent dyadic pattern is the sequence of a Duchenne smile of one person followed by a Duchenne smile of the other person at a similar time interval $[9,14]$. Repetitive dyadic sequence patterns can be understood as indicators of "interactive affective enmeshment" [14], and as a consequence, of interactive emotion regulation.

For a better understanding of the method, the results of the THEME analysis for therapy 01-2 are presented in Table 1 as an example.

\section{Insert Tab. 1}

During the first session of therapy A four repetitive sequence patterns (total number of patterns) were detected by THEME, one monadic pattern of the patient (No. 1), one monadic pattern of the therapist (No. 2), and two dyadic patterns (No. 3 and No. 4). The monadic 
pattern of the patient consisted of sadness-Pat $\rightarrow$ disgust-Pat and occured five times with an average time interval of 0.4 seconds between sadness and disgust. The most frequent pattern was the dyadic pattern of Dsmile-Ther $\rightarrow$ Dsmile-Pat (Duchenne smile of therapist followed by Duchenne smile of patient), which occured eight times.

Merten [14] called patterns which include affects of the same valence "reciprocal", and those including affects of different valence "compensatory". For example, a pattern of Dsmile-Ther $\rightarrow$ Dsmile-Pat would be a "reciprocal-positive" dyadic pattern, a pattern of anger-Pat $\rightarrow$ anger-Ther a "reciprocal-negative" dyadic pattern, and a pattern of Dsmile-Pat $\rightarrow$ anger-Ther (or vice versa) a compensatory pattern. The number and frequency of occurrence of the dyadic repetitive sequence patterns are taken as indicators for interactive affective enmeshment in this study.

\section{Results}

In the following, the results of the analysis of repetitive sequence patterns in facial affective behaviour of patients and their therapists in each first session are presented. The sequence patterns were determined by means of THEME. Table 2 contains descriptive data of the sequence patterns.

\section{Insert Tab. 2}

Numbers as well as the frequencies of occurrence of repetitive patterns show large ranges and high standard deviations. Dyadic patterns consisting of negative affects were rare and therefore are not listed in Table 2 and not considered separately in further analysis.

Patients responded to Duchenne smiles of their therapist significantly more often with a Duchenne smile, than vice versa (Wilcoxon-Test, $\mathrm{p}=.005$, 2-tailed), which confirms hypothesis 1 . 
It was assumed (Hypothesis 2) that the number of dyadic repetitive sequence patterns as well as the frequency of their occurrence correlates positively with symptomatic burden. Due to the fact that the number of monadic and dyadic patterns correlate significantly positively with the frequency of facial affective expressions of the patients (patients monadic pattern $+.57^{* *}$, dyadic pattern $+.49 *$, Spearman-Rho, 2-tailed), the data on sequence patterns were relativised on the frequency of primary affects and of Duchenne smiles of the patients, respectively. The statistical calculations underlying the following results are based on these relativised data.

The two monadic variables of patients (number and frequency of occurrence) correlate significantly positively with each other (Spearman-Rho $+.95^{* *}$ ) but not with the dyadic variables. All dyadic variables correlate significantly positively with each other (SpearmanRho varying from $+.55^{*}$ to $\left.+.96^{* *}\right)$. The total scores of the questionnaires show significant positive inter-correlations (Spearman-Rho): SCL-90R correlate with FBL $\left(+.71^{* *}\right)$, with STAI $\left(+.75^{* *}\right)$, and with BSQ $\left(+.78^{* *}\right)$, FBL correlate with ACQ $\left(+.57^{*}\right)$ and with BSQ $(+.89 * *)$, and ACQ correlate with BSQ $(+.59 *)$.

Table 3 shows the correlations between sequence patterns and symptom-variables.

\section{Insert Tab. 3}

Dyadic patterns highly correlate with measures of symptomatic burden (Table 3). The number of dyadic patterns as well as their frequency of occurrence correlates highly positively with the total score of the FBL and the BSQ, including both dyadic smile patterns (Dsmile-Pat $\rightarrow$ Dsmile-Ther and Dsmile-Ther $\rightarrow$ D-smile-Pat); additionally the latter shows a tendency to correlate with the ACQ. No significant correlations were found with the Global Severity Index of the SCL-90R and trait anxiety of the STAI. The dyadic sequence patterns mainly show correlations with physical symptoms. This is supported by an examination of SCL-90R and FBL sub-scales: While nearly all sub-scales of the FBL show significant correlations or a tendency to it, such correlations with the SCL-90R-scales are only found for the sub-scale 
somatisation. Thus, a stronger interactive enmeshment between patient and therapist is found in the sessions with patients with more severe physical symptoms.

\section{Discussion}

The number and frequency of occurrence of repetitive dyadic sequence patterns, considered as indicators of "interactive affective enmeshment" [14], shows significant positive correlations with the severity of symptomatic burden, especially with scales of physical symptoms. This is valid for dyadic patterns in general (independent of the specific facial affects involved), and especially for the dyadic repetitive patterns of Duchenne smile, the "felt smile" responded to by "felt smile". The more severe the patients' symptomatic burden, and in particular, the more they suffered from physical symptoms, the more often did these dyadic patterns occur. The overall higher interactive enmeshment of more severe symptomatically burdened patients could be understood as a diagnosis-overlapping finding: This result corresponds with the general clinical conception of a higher use of interactive affect regulation strategies of severely disturbed patients. The dominance of the Duchenne smile in dyadic repetitive sequence patterns, however, might be seen as an indicator for a panic-specific dynamic, in the sense, that patients with severe panic disorder try to establish a positive, "secure" and "harmonious" relationship interactively with their therapists to a considerable extent.

The dyadic repetitive patterns of Duchenne smiles were characterised by the fact that therapists responded to smile offers of their patients with smiles significantly less often than patients to those of their therapists; this can be understood as a manifestation of the interactive affective abstinence on the part of the therapists. However, the more severe symptomatic burden of patients, the more often did therapists respond to the smile offers of their patients, letting themselves be "dragged into" such interactive affective enmeshment to some extent. Possibly they could not resist the "interactive pressure" of these patients; at the same time, it 
is also likely that, due to a conscious or preconscious diagnosis [2], they allowed such an enmeshment because of the impression, that these patients needed more affective support. It is quite likely that interactive affective enmeshment is the result of both, pressure from the patients and a possibly unconscious desire of therapists to offer greater affective support to patients suffering from more severe panic disorder. But in some cases, negative affective enmeshment has also been observed which would offer more support to the first interpretation.

Limitations arise from the small sample and the lack of a comparison-group, hence the question if the results are disorder-specific or more general cannot be answered. For this reasons, the results of the presented study have to be regarded as preliminary. Nonetheless, these findings do indicate that dyadic affective indicators of symptomatic burden can be found also within distinct homogeneous diagnostic groups. Furthermore, these indicators are in good agreement with the described relationship dynamic of the investigated group of panic disorders.

\section{References}

1. Ekman P: An Argument for Basic Emotions. Cognition \& Emotion 1992, 6 (3/4), 169200.

2. Krause R: Allgemeine psychoanalytische Krankheitslehre. Bd. 1: Grundlagen. Stuttgart, Berlin: Kohlhammer, 1997.

3. Magai $\mathrm{C}, \mathrm{McF}$ adden $\mathrm{SH}$ : The role of emotions in social and personality development: History, theory, and research. New York: Plenum, 1995.

4. Bänninger-Huber E: Mimik - Übertragung - Interaktion. Die Untersuchung affektiver Prozesse in der Psychotherapie. Göttingen: Huber, 1996.

5. Steimer-Krause E, Krause R, Wagner G: Prozesse der Interaktionsregulierung bei schizophren und psychosomatisch erkrankten Patienten - Studien zum mimischen 
Verhalten in dyadischen Interaktionen. Zeitschrift für Klinische Psychologie 1990, 19 (1), 32-49.

6. Ellgring H, Gaebel W: Facial expression in schizophrenic patients. In: A. Beigel, J.J. Lopez, E. Costa \& J.A. Silva (Eds.), Past, present, and future of psychiatry, Vol. 1., pp. 435-439, 1994.

7. Merten J: Affekte und die Regulation nonverbalen, interaktiven Verhaltens. Bern: Peter Lang, 1996.

8. Steimer-Krause E: Übertragung, Affekt und Beziehung. Bern: Peter-Lang, 1996.

9. Schwab F: Affektchoreographien. Eine evolutionspsychologische Analyse von Grundformen mimisch-affektiver Interaktionsmuster. Berlin: dissertation.de, 2001.

10. Merten J, Brunnhuber S: Facial expression and experience of emotions in psychodynamic interviews with patients suffering from a pain disorder. Psychopathology 2004, 37, 266-271.

11. Rasting M, Brosig B, Beutel M: Alexithymic characteristics and patient-therapistinteraction: a video-analysis of facial affect display. Psychopathology, 2005, 38, 105111.

12. Krause R: Allgemeine psychoanalytische Krankheitslehre. Bd. 2: Modelle. Stuttgart, Berlin: Kohlhammer, 1998.

13. Fonagy P, Gergely G, Jurist EL, Target M: Affect regulation, mentalization, and the development of the self. New York: Other Press, 2002.

14. Merten J: Beziehungsregulation in Psychotherapien. Maladaptive Beziehungsmuster und der therapeutische Prozess. Stuttgart: Kohlhammer, 2001.

15. Rasting M, Beutel M: Dyadic affective interactive patterns in the intake interview as a predictor of outcome. Psychotherapy Research, 2005, 15 (3): 188-198.

16. Milrod BL, Busch FN, Cooper AM, Shapiro T: Manual of Panic-Focused Psychodynamic Psychotherapy. Washington: American Psychiatric Press, 1997. 
17. Scheibe G, Tress W, Reister G: Psychoanalytische Modellvorstellungen und die DSMIV-Klassifikation von Angststörungen: Widerspruch oder Ergänzung? Zsch. Psychosom. Med., 1997, 43, 138-152.

18. König K: Angst und Persönlichkeit. Göttingen: Vandenhoeck \& Ruprecht, 1981.

19. Krause R, Lütolf R: Facial indicators of transference processes within psychoanalytic treatment. In Dahl H, Kächele H, Thomä H (eds.) Psychoanalytic Process Research Strategies (pp257-272). Berlin: Springer, 1988.

20. Benecke C, Krause R: Facial-affective relationship-offers of patients with panicdisorder. Psychotherapy Research, 2005, 15 (3), 178-187.

21. Hiller W, Zaudig M, Mombour W / WHO: ICD-10-Checklisten. Bern: Huber, 1995.

22. Franke GH: SCL-90R. Die Symptom-Checkliste von Derogatis. Göttingen: Beltz, 1995.

23. Fahrenberg J: Freiburger Beschwerden-Liste. Göttingen: Hogrefe, 1994.

24. Laux L, Glanzmann P, Schaffner P, Spielberger CD: STAI - Das State-TraitAngstinventar. Göttingen: Beltz, 1981.

25. Ehlers A, Margraf J, Chambless D: Fragebögen zu körperbezogenen Ängsten, Kognitionen und Vermeidung. Weinheim: Beltz, 1993.

26. Friesen WV, Ekman P: EMFACS-7. Unveröffentlichtes Manual, 1984.

27. Ekman P, Friesen WV: Facial Action Coding System (FACS): Manual. Palo Alto: Consulting Psychological Press, 1978.

28. Ekman P, Friesen WV, Hagar JC: Facial Action Coding System (FACS): Manual. Second Edition. Salt Lake City: Research Nexus eBook, 2002.

29. Magnusson MS: THEME USER'S MANUAL: with notes on theory, model and pattern detection method. Human Behavior Laboratory, Unitversity of Iceland, 1993. 
30. Magnusson MS: Hidden real-time patterns in intra- and inter-individual behaviour: description and detection. European Journal of Psychological Assessment, 1996, 12 (2), 112-123.

31. Sayette MA, Cohn JF, Wertz JM, Perrot MA, Parrott DJ: A psychometric evaluation of the Facial Action Coding System for assessing spontaneous expression. J Nonverbal Behavior, 2001, 25, 167-185. 
Tab. 1: Repetitive sequence patterns in therapy A, in the first session (THEME, p > .01) ("Pat” = patient; "Ther" = therapist; “Dsmile”= Duchenne smile)

\begin{tabular}{|c|c|c|c|}
\hline \multicolumn{4}{|c|}{ Repetitive Sequence Patterns in Therapy A, session 1} \\
\hline No. & pattern & frequency of & mean intervals \\
\hline & & occurrence & in seconds \\
\hline 1 & sadness-Pat $\rightarrow$ disgust-Pat & 5 & 0.4 \\
\hline 2 & Dsmile-Ther $\rightarrow$ disgust-Ther & 5 & 11.6 \\
\hline 3 & Dsmile-Ther $\rightarrow$ Dsmile-Pat & 8 & 0.9 \\
\hline 4 & anger-Pat $\rightarrow$ anger-Ther & 5 & 0.4 \\
\hline
\end{tabular}


Tab. 2: Repetitive sequence pattern in the first session $(n=20$; relative to duration of session: converted uniformly to 50 min; values rounded; "Pat” = patient; “Ther” = therapist; “Dsmile”= Duchenne smile).

Repetitive Sequence Patterns in the first session; 20 Dyads

\begin{tabular}{llccc} 
& & Mean & SD & Range \\
Number & monadic-Pat, all affects & 1.4 & 1.6 & $0-4.6$ \\
& dyadic, all affects & 3.9 & 3.8 & $0-12.7$ \\
Frequency of & monadic-Pat, all affects & 7.4 & & $0-24.5$ \\
occurrence & dyadic, all affects & 25.9 & 8.9 & $0-87.5$ \\
& Dsmile-Pat $\rightarrow$ Dsmile-Ther & 3.4 & 26.9 & $0-16.7$ \\
& Dsmile-Ther $\rightarrow$ Dsmile-Pat & 9.9 & 4.4 & $0-26.5$ \\
\hline
\end{tabular}


Tab. 3: Correlations between sequence patterns and symptomatic burden ( $n=15$; except for SCL-90R n=13; nonparametric correlation Spearman-Rho, 2-tailed, values rounded; ${ }^{+}=\mathrm{p}<.10,{ }^{*}=\mathrm{p}<.05, * *=\mathrm{p}<.01$; “Pat” $=$ patient; “Ther” = therapist; “Dsmiles”= Duchenne smiles).

$$
\begin{array}{ccc}
\text { Number of patterns, } & \text { Frequency of occurrence, } & \text { Frequency of occurrence, } \\
\text { all affects } & \text { all patterns } & \text { dyadic patterns of Dsmiles }
\end{array}
$$

\begin{tabular}{|c|c|c|}
\hline Symptomatic & (adjusted to patient's & (adjusted to patient's \\
\hline
\end{tabular}

burden

activity)

activity)

Dsmiles)

Total scores

monadic Pat dyadic

monadic Pat

dyadic

Pat $\rightarrow$ Ther

Ther $\rightarrow$ Pat

SCL-90R

FBL

$+.72 * *$

$+.71 * *$

$+.66^{* *}$

$+.69 * *$

STAI

ACQ

$+.50^{+} \quad+.49^{+}$

BSQ

$+.69 * *$

$+.65 *$

$+.63 *$

$+.70 * *$ 